\title{
ENDOSCOPY
}

\section{NOTES reveals obscure origin of ascites}

A new study by Bai et al. has found that transgastric NOTES (natural orifice transluminal endoscopic surgery), in combination with biopsy, provided a diagnosis in $92.3 \%$ of patients with ascites of unknown origin, for which previous attempts fell short of a clear diagnosis.

NOTES is a minimally invasive technique that aims to reduce surgical trauma by using natural orifices rather than external incisions to gain access to the peritoneal cavity. In the past few years NOTES has transitioned from the laboratory to the clinic. Ascites can be evaluated using many tools, including imaging techniques and laboratory tests. Despite the array of assessment methods available, a proportion of patients are unable to receive a clear diagnosis of the underlying cause of their ascites. Bai and colleagues decided to investigate whether NOTES could be advantageous in this clinical setting.

Drainage and biopsy were all performed via endoscopic access through the stomach wall. Biopsy material was successfully collected from all 78 patients, with 72 patients receiving a clear diagnosis. Various pathologies were diagnosed, $54 \%$ (39 of 72 ) of which were malignant tumours.

The major concern with NOTES is post-surgical complications; however, in this study no adverse events occurred immediately after surgery. At the 1.5 year follow-up, no serious adverse events were reported, but minor adverse events included transient abdominal pain, slight distension and transient fever. The study highlights the safety of transgastric NOTES in patients with ascites, but the authors point out the need for direct comparisons with established techniques, such as laparoscopy, before it can become routine.

Gillian Patman

Original article Bai, Y. et al. Role of transgastric natural orifice transluminal endoscopic surgery in the diagnosis of ascites of unknown origin (with videos). Gastrointest. Endosc. doi:10.1016/j.gie.2014.03.025 